\section{Differential diagnostic factors of type 1 and type 2 myocardial infarction in patients with elevated cardiac troponin levels}

\author{
Youngho Seo', Jinhui Paik', Seunglyul Shin', Ahjin Kim², Soo Kang' \\ 'Department of Emergency Medicine, Inha University College of Medicine, Incheon, Korea \\ ${ }^{2}$ Department of Hospital Medicine, Inha University College of Medicine, Incheon, Korea
}

Objective Emergency physicians experience difficulty in determining the disposition of patients with elevated troponin I levels using emergency room tests. In this study, we aimed to investigate factors that could discriminate between the occurrence of type 1 myocardial infarction (T1MI) and type 2 myocardial infarction (T2MI) in patients with elevated troponin I levels.

Methods Patients admitted to the emergency department between January 1, 2017 and June 30, 2017 with elevated troponin I levels who underwent subsequent cardiac biomarker testing were included. Samples for baseline blood tests, such as cardiac biomarker levels, were collected within approximately 10 minutes of admission. Electrocardiogram, transthoracic echocardiography, and percutaneous coronary intervention results were retrospectively examined via patient report and chart reviews.

Results During the study period, 169 of 234 (72\%) patients were diagnosed with T2Ml and 65 $(28 \%)$ were diagnosed with T1MI. Among various factors, typical chest pain (odds ratio [OR], 4.40; 95\% confidence interval $[\mathrm{Cl}], 1.46$ to $13.24 ; \mathrm{P}=0.008)$, high troponin I levels $(\mathrm{OR}, 1.50$; $95 \% \mathrm{Cl}, 1.19$ to $1.90 ; \mathrm{P}<0.001)$, high cholesterol $(\mathrm{OR}, 1.01 ; 95 \% \mathrm{Cl}, 1.00$ to $1.02 ; \mathrm{P}=0.008)$, and low $\mathrm{D}$-dimer levels $(\mathrm{OR}, 0.87 ; 95 \% \mathrm{Cl}, 0.77$ to $0.98 ; \mathrm{P}=0.027)$ were significantly associated with $\mathrm{T} 1 \mathrm{Ml}$ incidence.

Conclusion Our findings in this study indicate that typical chest pain, high levels of troponin I and cholesterol, and low levels of D-dimer were associated with the diagnosis of T1MI. Further studies are suggested to determine the cut-off values for accurate diagnosis of T1MI in the ED.

Keywords Troponin I; Myocardial infarction; Acute coronary syndrome

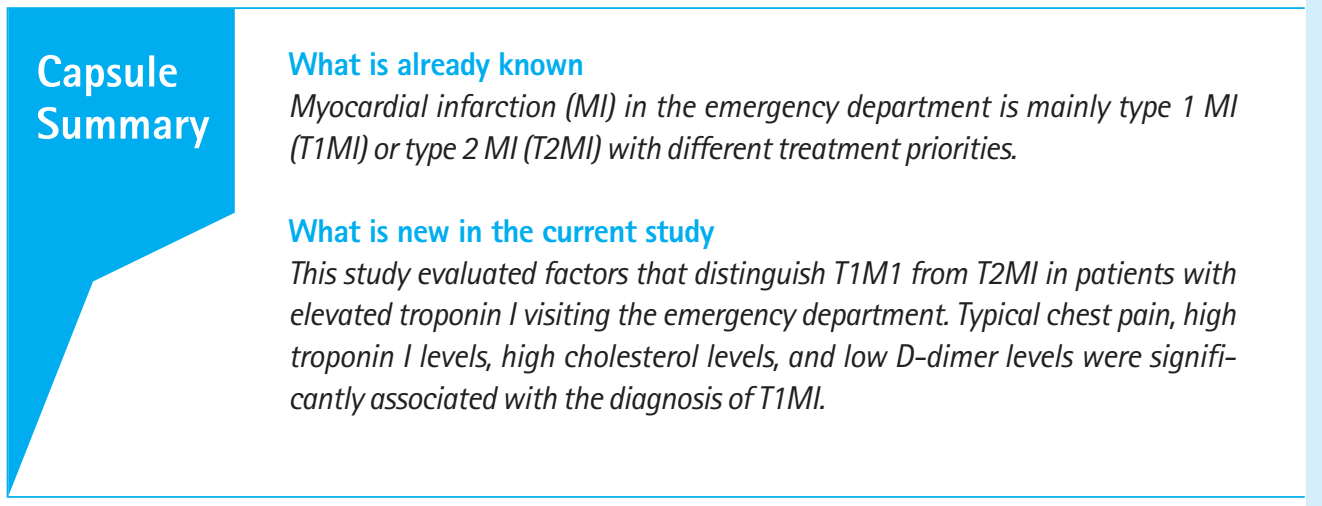

elSSN: 2383-4625

Received: 10 June 2019

Revised: 17 July 2019

Accepted: 22 July 2019

Correspondence to: Soo Kang Department of Emergency Medicine, Inha University College of Medicine, 27 Inhang-ro, Jung-gu, Incheon 22332, Korea

E-mail: skang790131@inhauh.com ORCID

http://orcid.org/0000-0003-0800-1350

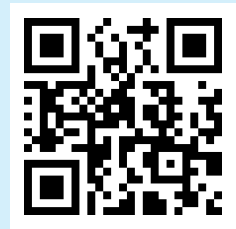

How to cite this article: Seo Y, Paik J, Shin S, Kim A, Kang S. Differential diagnostic factors of type 1 and type 2 myocardial infarction in patients with elevated cardiac troponin levels. Clin Exp Emerg Med 2020;7(3):213-219. https://doi. org/10.15441/ceem. 19.049

This is an Open Access article distributed under the terms of the Creative Commons Attribution Non-Commercial License (https:// creativecommons.org/licenses/by-nc/4.0/). 


\section{INTRODUCTION}

The early diagnosis of myocardial infarction (MI) in patients admitted to the emergency department is important for their effective treatment and a good prognosis. According to data reported by the Korea National Statistical Office in 2017, heart disease is the second leading cause of death after malignant neoplasms, and its incidence is on the rise. ${ }^{1} \mathrm{Ml}$ refers to myocardial cell damage caused by impaired blood flow to the heart due to coronary artery spasm or obstructive thrombosis and the resultant reduced myocardial oxygen and nutrient supply. ${ }^{2}$ In general, $\mathrm{Ml}$ is diagnosed according to clinical symptoms, and the results of electrocardiogram (ECG) and cardiac biomarker tests. However, because atypical or asymptomatic MI can occur, most emergency centers routinely and selectively perform cardiac biomarker testing in high-risk patients with heart disease. According to the third universal definition of MI updated in 2012, MI is classified into five types based on pathology. ${ }^{3}$ Type $1 \mathrm{MI}(\mathrm{T} 1 \mathrm{MI})$ is classified as spontaneous MI related to coronary artery disease, caused by atherosclerotic plaque rupture and thrombosis. Type $2 \mathrm{MI}$ (T2MI) is classified as $\mathrm{Ml}$ related to an imbalance between myocardial oxygen supply and demand. Type $3 \mathrm{MI}$ (T3MI) is classified as MI resulting in sudden death, which cannot be diagnosed without autopsy. Type 4 and $5 \mathrm{MI}$ (T4MI, T5MI) are classified as MI related to percutaneous coronary intervention (PCI) and coronary artery bypass grafting performed by physicians, respectively. The differential diagnosis of $\mathrm{T} 1 \mathrm{MI}$ and non-type $1 \mathrm{MI}$ (T2MI-T5MI) is important when choosing a treatment option for $\mathrm{Ml}$ among $\mathrm{PCl}$ or drug therapy. ${ }^{4.5}$ It is difficult for emergency physicians to differentiate between T1MI and T2MI-T5MI when cardiac biomarker test results reveal elevated myocardial biomarker levels. In this study, we investigated clinical features and risk factors that could be used for the differential diagnosis of $\mathrm{T} 1 \mathrm{Ml}$ and $\mathrm{T} 2 \mathrm{Ml}$ in patients with elevated troponin I levels who were admitted to the emergency department. ${ }^{6}$

\section{METHODS}

\section{Study setting and population}

This study was conducted in the emergency center of a tertiary training hospital with 925 beds, where approximately 60,000 patient visits each year. Subjects in this study included patients $\geq 16$ years of age who visited this hospital between January 1, 2017 and June 30, 2017, and underwent troponin I assays. Patients with ST segment elevation MI on their initial ECG or those who experienced cardiac arrest at the time of ED arrival were excluded from the study. Patients who failed to undergo follow-up tests, either because they were voluntarily discharged from the emergency department or transferred to another hospital, or died before tests could be carried out (except for myocardial biomarker tests), were also excluded. The institutional review board of the Inha University Hospital approved this study (2019-05-023). Informed consent was waived for this study due to retrospective design.

\section{Data collection}

Demographic, clinical, and laboratory data of the subjects were collected from the related electronic medical records. Based on the basic information of the admitted patients, age and sex were identified, and height and weight were measured. Other demographic data were collected by questionnaire. History of smoking, cardiac symptoms, familial history of coronary artery disease, comorbidities (diabetes mellitus, hypertension, or hypercholesterolemia), and a history of coronary revascularization, $\mathrm{Ml}$, stroke, and peripheral arterial occlusive disease were determined by physicians involved in the initial diagnosis and the nurses in charge. ECG findings were divided into three categories following the history, electrocardiogram, age, risk factors, and troponin (HEART) score: normal, non-specific repolarization (ST-T wave changes; abnormal but non-ischemic changes), and significant ST-depression. Transthoracic echocardiography (TE) and coronary angiography findings were referred to in the official reports.

Chest pain in patients was defined as either typical pain or atypical pain. Typical chest pain referred to pressure or squeezing pain on the center or left-sided chest, radiating to the jaw or throat or arm, with sweating or clamminess. Atypical chest pain referred to pain on the right-sided chest, radiating to the back or worsened on inspiration or palpitation. Patients were categorized as experiencing typical pain, both typical and atypical pain, or atypical pain only. ${ }^{8}$

\section{Blood measurement and troponin assay}

Venous blood samples were collected from patients within approximately 10 minutes of arrival at the emergency department. Laboratory test results, including white blood cell count, platelet count, neutrophil count, and levels of hemoglobin, blood urea nitrogen, creatinine, total bilirubin, aspartate aminotransferase, alanine aminotransferase, cholesterol, potassium, chloride, N-terminal proB-type natriuretic peptide (NT pro-BNP), D-dimer, and C-reactive protein (CRP), were retrospectively studied by reviewing laboratory reports. Levels of troponin I, a cardiac biomarker, were tested in blood samples collected in EDTA (ethylenediaminetetraacetic acid) tubes (containing heparin) from the patient's vein within an average of 10 minutes. Each blood tube was sent 
to the clinical hematology laboratory of Inha University Hospital for centrifugation before analysis. Measurements were conducted according to the manufacturer's instructions by using the Elecsys cobas e 411 system (Roche Diagnostic, Basel, Switzerland).

\section{Outcome variables}

Patients who showed changes from previous findings following $\mathrm{PCl}$ performed after admission, those with suspected ischemic heart disease based on TTE findings, and those with suspected ischemic heart disease during the one-month outpatient followup were defined as the T1Ml group. --> In contrast, patients who demonstrated no changes from previous findings following $\mathrm{PCl}$, those not suspected of having ischemic heart disease on TE findings, and those not suspected of having ischemic heart disease during the one-month outpatient follow-up were defined as the T2Ml group.

\section{Statistical analysis}

Continuous variables that are normally distributed, as determined by normality testing, are expressed as mean \pm standard deviation. Continuous variables that are not normally distributed are expressed as median (interquartile range, IQR) after analysis using the Mann-Whitney U-test. Categorical variables were analyzed using the chi-squared test. Multivariate logistic regression analysis was performed after adjusting for confounding variables in the univariate analysis. All data were analyzed using IBM SPSS Statistics ver. 19.0 (IBM Corp., Armonk, NY, USA).

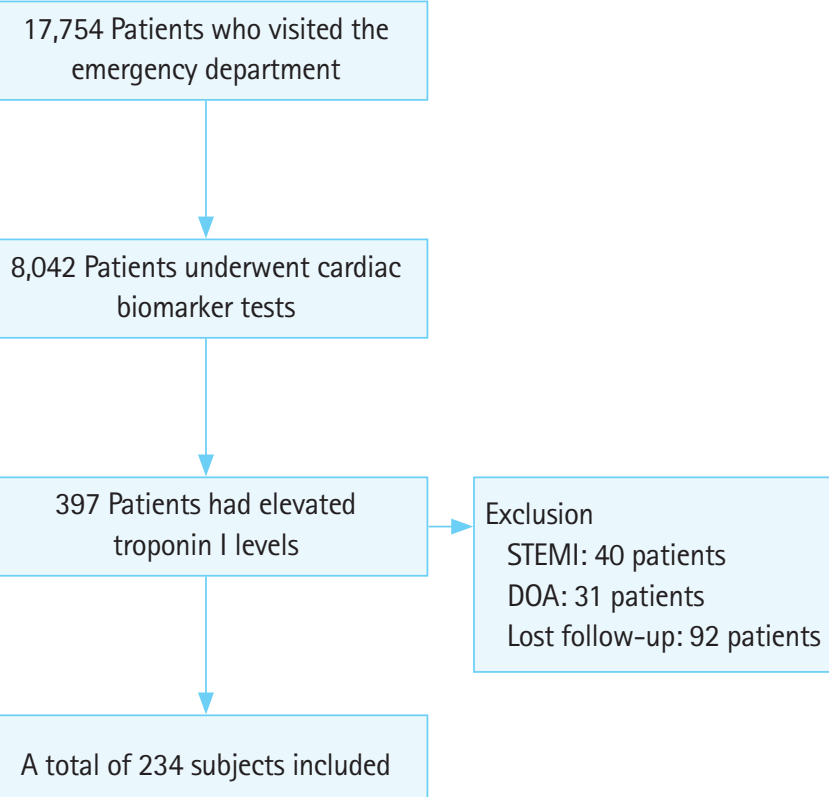

Fig. 1. Flow chart of patients included in this study. STEMI, ST segment elevation myocardial infarction; DOA, dead on arrival.

\section{RESULTS}

\section{Characteristics of the patients}

Of the 17,754 patients who visited the emergency department during the study period, 8,042 (45.3\%) underwent cardiac biomarker tests due to chest pain and suspected acute heart disease or old age. Of these 8,042 patients, 397 had elevated troponin I levels (normal range, $0.000-0.160 \mathrm{ng} / \mathrm{mL}$ ). Of these 397 patients, 40 were diagnosed with ST segment elevation MI on their initial ECG, 31 were in cardiac arrest at the time of ED arrival, and 92 were not followed up as they were either voluntarily discharged from the emergency department, transferred to another hospital, or died before testing. These patients were excluded from the study. Therefore, a total of 234 subjects were ultimately included in the analysis (Fig. 1).

\section{Univariate analysis: clinical and electrocardiogram findings}

Baseline patient characteristics are shown in Table 1. Of the 234 subjects included, 169 (72\%) were diagnosed with T2Ml and 65 (28\%) with T1MI. The median age of those with T2MI was 69 years (IQR, 53 to 79 years), whereas the median age of those with T1MI was 73 years (IQR, 58 to 78 years), with no significant difference in age between the two groups $(P=0.216)$. The proportion of men with T2MI was 51.5\% (87 of 169) and the proportion of men with $\mathrm{T} 1 \mathrm{Ml}$ was $69.2 \%$ (45 of 65), whereas the proportion of women with $\mathrm{T} 2 \mathrm{MI}$ was $48.5 \%$ (82 of 169) and the proportion

Table 1. Comparison of patient baseline characteristics

\begin{tabular}{lcccc}
\hline Variable & $\begin{array}{c}\text { All } \\
(\mathrm{n}=234)\end{array}$ & $\begin{array}{c}\text { T2MI } \\
(\mathrm{n}=169)\end{array}$ & $\begin{array}{c}\text { T1MI } \\
(\mathrm{n}=65)\end{array}$ & P-value \\
\hline Age $(\mathrm{yr})$ & $69(54-78)$ & $69(53-79)$ & $73(58-78)$ & 0.216 \\
Sex, male & $132(56.4)$ & $87(51.5)$ & $45(69.2)$ & $0.018^{*}$ \\
Medical history & & & & \\
$\quad$ Diabetes mellitus & $70(29.9)$ & $51(30.2)$ & $19(29.2)$ & 1.000 \\
Hypertension & $114(48.7)$ & $77(45.6)$ & $37(56.9)$ & 0.144 \\
Smoking & $67(28.6)$ & $43(25.4)$ & $24(36.9)$ & 0.106 \\
Hyperlipidemia & $26(11.1)$ & $18(10.7)$ & $8(12.3)$ & 0.817 \\
FHx of CAD & $2(0.9)$ & $1(0.6)$ & $1(1.5 \%)$ & 1.000 \\
Hx of CAD & $26(11.1)$ & $16(9.5)$ & $10(15.4)$ & 0.245 \\
Hx of MI & $29(12.4)$ & $16(9.5)$ & $13(20.0)$ & $0.044^{*}$ \\
Hx of stroke & $32(13.7)$ & $20(11.8)$ & $12(18.5)$ & 0.205 \\
Hx of PAOD & $6(2.6)$ & $5(3.0)$ & $1(1.5)$ & 0.681 \\
Obesity (BMI > 25 kg/m²) & $16(6.8)$ & $11(6.5)$ & $5(7.7)$ & 0.775 \\
\hline
\end{tabular}

Values are presented as median (interquartile range) or number (\%). T2MI, type 2 myocardial infarction; T1MI, type 1 myocardial infarction; $F H x_{\text {, }}$ family history; $\mathrm{CAD}$, coronary artery disease; $\mathrm{Hx}$, history; $\mathrm{Ml}$, myocardial infarction; PAOD, peripheral arterial occlusive disease; BMI, body mass index. ${ }^{*} P<0.05$, significant change from baseline values. 
of women with $\mathrm{T} 1 \mathrm{MI}$ was $30.8 \%$ (20 of 65 ), resulting in a significantly higher proportion of men with $\mathrm{T} 1 \mathrm{Ml}(\mathrm{P}=0.018)$. The proportion of T2Ml patients with a history of $\mathrm{Ml}$ was 9.5\% (16 of 169 subjects). History of MI was a significant factor predictive of T1MI $(P=0.044) ; 20.0 \%$ of patients with T1MI had a history of MI (13 of 65 subjects). Regarding other risk factors, there was no significant association found between these conditions and the presence of diabetes mellitus $(P=1.000)$, hypertension $(P=0.144)$, or hyperlipidemia $(P=0.817)$ among underlying conditions; history of smoking $(P=0.106)$ among social history; cardiovascular disease $(P=1.000)$ and body mass index (BMl, obesity defined as a $\left.\mathrm{BMI} \geq 25 \mathrm{~kg} / \mathrm{m}^{2}\right)(\mathrm{P}=0.775)$ among family history; and history of previous $\mathrm{PCl}(\mathrm{P}=0.245)$, cerebrovascular disease $(P=0.205)$ or peripheral arterial occlusive disease $(P=0.681)$ among medical history (Table 1). T1MI was more likely in patients with typical symptoms, and T2MI was more likely in patients with atypical symptoms (T1MI $n(\%)$ vs. T2MI $n(\%)$ : typical symptoms $=21$ $(72.4 \%)$ vs. $8(27.6 \%)$, both typical and atypical symptoms $=11$ $(42.3 \%)$ vs. $15(57.7 \%)$, and atypical symptoms $=33(18.4 \%)$ vs.
$146(81.6 \%), P<0.001)$. There was no significant difference in ECG findings between the T1MI and T2MI groups (T1MI $n$ [\%] vs. T2MI $n$ [\%]: normal or nonspecific ST-T wave change $=13$ [18.8\%] vs. 56 [81.2\%], abnormal but not diagnostic ischemia $=37$ [31.4\%] vs. 81 [68.6\%], finding of infarction or ischemia not old $=15$ [31.9\%] vs. $32[68.1 \%], P=0.090)$.

\section{Univariate analysis: laboratory findings}

Laboratory findings are shown in Table 2 . In blood tests involving cardiac biomarkers, there were significant differences between the T2MI and T1Ml groups, specifically in troponin I levels (median 0.34 [IQR $0.22-0.61$ ] vs. 0.94 [IOR 0.26-2.69], P<0.001), creatine kinase muscle and brain (CK-MB) levels (median 5.10 [IOR 2.80-11.00] vs. 8.00 [IOR 4.2-27.30], $\mathrm{P}=0.001), \mathrm{CK} / \mathrm{CK}-\mathrm{MB}$ ratio (median 2.96 [IQR 1.39-5.53] vs. 4.65 [IQR 2.66-8.04], $\mathrm{P}=0.001$ ), and cholesterol levels (median 141.00 [IQR 111-165.50] vs. 174.00 [IQR 138-201], $P \leq 0.001$ ) (Table 2). These results show these markers were significantly higher in the T1Ml group. In addition, D-dimer levels (median 2.70 [IOR 0.93-6.36] vs 1.14 [IOR 0.50-

Table 2. Comparison of laboratory findings between T2Ml and T1MI

\begin{tabular}{|c|c|c|c|c|c|c|c|}
\hline & \multicolumn{2}{|c|}{ All $(n=234)$} & \multicolumn{2}{|c|}{ T2MI $(n=169)$} & \multicolumn{2}{|c|}{ T1MI $(n=65)$} & \multirow{2}{*}{ P-value } \\
\hline & Median & IQR & Median & IQR & Median & IQR & \\
\hline \multicolumn{8}{|l|}{ Cardiac enzyme } \\
\hline Tnl (ng/mL) & 0.38 & $0.23-1.03$ & 0.34 & $0.22-0.61$ & 0.94 & $0.26-2.69$ & $<0.001^{* * *}$ \\
\hline CK (IU/L) & 172.50 & $88-342.75$ & 163.00 & $79.50-325.50$ & 194.00 & $117-915$ & 0.099 \\
\hline CK-MB (ng/mL) & 5.50 & $2.98-12.88$ & 5.10 & $2.80-11.00$ & 8.00 & $4.2-27.30$ & $0.001^{* *}$ \\
\hline CK/CK-MB & 3.72 & $1.62-6.25$ & 2.96 & $1.39-5.53$ & 4.65 & $2.66-8.04$ & $0.001^{* *}$ \\
\hline NT-pro BNP (pg/mL) & 7,093 & $1,736.25-25,361.75$ & $5,962(n=93)^{a)}$ & $1,694.50-25,506.50$ & $8,502(n=24)^{a)}$ & $1,808-27,931.50$ & 0.593 \\
\hline $\mathrm{D}$-dimer $(\mu \mathrm{g} / \mathrm{mL})$ & 2.28 & $0.70-5.36$ & 2.70 & $0.93-6.36$ & 1.14 & $0.50-2.74$ & $<0.001^{* * *}$ \\
\hline \multicolumn{8}{|c|}{ Complete blood cell count } \\
\hline WBC $(1,000 / \mu \mathrm{L})$ & 9.78 & $7.10-13.64$ & 9.92 & $7.12-14.11$ & 9.24 & $7.05-12.62$ & 0.200 \\
\hline $\mathrm{Hb}(\mathrm{g} / \mathrm{dL})$ & 12.40 & $10.30-14.10$ & 12.15 & $10.40-13.60$ & 13.35 & $10.03-14.85$ & 0.099 \\
\hline PIt $(1,000 / \mu \mathrm{L})$ & 206.50 & $149-265$ & 196.50 & $143.50-261.50$ & 220.00 & $172-266.75$ & 0.194 \\
\hline Neutrophil (\%) & 78.70 & $68.85-88$ & 82.15 & $72.13-88.38$ & 71.70 & $60.45-81.55$ & $<0.001^{* * *}$ \\
\hline $\mathrm{CRP}(\mathrm{mg} / \mathrm{dL})$ & 1.98 & $0.34-7.80$ & 2.37 & $0.47-8.08$ & 0.72 & $0.16-11.82$ & $0.025^{*}$ \\
\hline BUN (mg/dL) & 21.55 & $13.68-38.93$ & 21.80 & $13.65-41.90$ & 19.60 & $13.60-28.50$ & 0.431 \\
\hline $\mathrm{Cr}(\mathrm{mg} / \mathrm{dL})$ & 1.11 & $0.81-2.23$ & 1.09 & $0.78-2.25$ & 1.15 & $0.86-1.79$ & 0.716 \\
\hline $\mathrm{Tb}(\mathrm{mg} / \mathrm{dL})$ & 0.60 & $0.40-0.90$ & 0.60 & $0.40-0.90$ & 0.50 & $0.35-0.80$ & 0.304 \\
\hline AST (IU/L) & 37 & $24-63$ & 38.00 & $24-67.50$ & 36.00 & $25.50-53.50$ & 0.682 \\
\hline ALT (IU/L) & 22 & $14-42.75$ & 25.00 & $14-50$ & 18.00 & $13.50-34$ & 0.072 \\
\hline $\mathrm{Na}(\mathrm{mEq} / \mathrm{L})$ & 137 & $133-139$ & 136.00 & $132-139$ & 137.00 & $134.50-139.50$ & 0.104 \\
\hline $\mathrm{K}(\mathrm{mEq} / \mathrm{L})$ & 4 & $3.70-4.63$ & 4.10 & $3.60-4.70$ & 4.00 & $3.80-4.40$ & 0.730 \\
\hline $\mathrm{Cl}(\mathrm{mEq} / \mathrm{L})$ & 99 & $95-102$ & 99.00 & $95-102$ & 100.00 & $97-103$ & 0.063 \\
\hline Cholesterol (mg/dL) & 145.50 & $114.75-181.25$ & 141.00 & $111-165.50$ & 174.00 & $138-201$ & $<0.001^{* * *}$ \\
\hline
\end{tabular}

T2MI, type 2 myocardial infarction; T1MI, type 1 myocardial infarction; IQR, interquartile range; Tnl, troponin I; CK, creatine kinase; MB, muscle and brain; NT-pro BNP, brain natriuretic peptide; WBC, white blood cell count; Hb, hemoglobin; Plt, platelet; CRP, C-reactive protein; BUN, blood urea nitrogen; Cr, creatine; Tb, total bilirubin; AST, aspartate aminotransferase; ALT, alanine aminotransferase; Na, sodium; K, potassium; $\mathrm{Cl}$, chloride.

${ }^{*} \mathrm{P}<0.05,{ }^{* *} \mathrm{P}<0.01,{ }^{* *} \mathrm{P}<0.001$, significant change from baseline values. ${ }^{\text {a) }}$ Missing value (n): T2MI 76 of $169(45 \%), \mathrm{T} 1 \mathrm{MI} 24$ of 65 (36.9\%). 
Table 3. Risk factors for type 1 myocardial infarction

\begin{tabular}{|c|c|c|c|c|}
\hline \multirow[b]{2}{*}{ Characteristics } & \multicolumn{2}{|c|}{ Univariate analysis } & \multicolumn{2}{|c|}{ Multivariate analysis } \\
\hline & $\begin{array}{l}\text { Unadjusted OR } \\
(95 \% \mathrm{Cl})\end{array}$ & P-value & $\begin{array}{l}\text { Adjusted OR } \\
(95 \% \mathrm{Cl})\end{array}$ & P-value \\
\hline Sex, male & $2.12(1.16-3.89)$ & $0.018^{*}$ & $1.86(0.82-4.21)$ & 0.136 \\
\hline \multicolumn{5}{|l|}{ Medical history } \\
\hline MI & $2.39(1.08-5.30)$ & $0.044^{*}$ & $2.32(0.85-6.36)$ & 0.101 \\
\hline $\mathrm{Tnl}(\mathrm{ng} / \mathrm{mL})$ & $1.41(1.18-1.69)$ & $<0.001^{* * *}$ & $1.50(1.19-1.90)$ & $<0.001^{* * *}$ \\
\hline CK/CK-MB & $1.07(1.01-1.15)$ & $0.036^{*}$ & $1.04(0.95-1.14)$ & 0.369 \\
\hline D-dimer $(\mu \mathrm{g} / \mathrm{mL})$ & $0.85(0.76-0.95)$ & $0.003^{* *}$ & $0.87(0.77-0.98)$ & $0.027^{*}$ \\
\hline $\mathrm{CRP}(\mathrm{mg} / \mathrm{dL})$ & $0.96(0.92-1.01)$ & 0.084 & $1.01(0.96-1.07)$ & 0.624 \\
\hline Cholesterol (mg/dL) & $1.01(1.01-1.02)$ & $<0.001^{* * *}$ & $1.01(1.00-1.02)$ & $0.008^{* *}$ \\
\hline Neutrophil (\%) & $0.97(0.95-0.99)$ & $0.001^{* * *}$ & $0.99(0.96-1.02)$ & 0.655 \\
\hline
\end{tabular}

$\mathrm{OR}$, odds ratio; $\mathrm{Cl}$, confidence interval; $\mathrm{MI}$, myocardial infarction; $\mathrm{Tnl}$, troponin I; $\mathrm{CK}$, creatine kinase; $\mathrm{MB}$, muscle and brain; CRP, C-reactive protein.

${ }^{*} \mathrm{P}<0.05,{ }^{* *} \mathrm{P}<0.01,{ }^{* *} \mathrm{P}<0.001$, significant change from baseline values.

2.74], $P<0.001$ ), CRP levels (median 2.37 [IQR 0.47-8.08] vs. 0.72 [IQR 0.16-11.82], $P=0.025$ ), and neutrophil counts (median 82.15 [IOR 72.13-88.38] vs. 71.70 [IQR 60.45-81.55], $\mathrm{P}<0.001$ ) were significantly higher in the T2MI group. There were no significant differences in the other blood test findings.

\section{Multivariate analyses}

The results of multivariate logistic regression analyses after controlling for confounding variables revealed statistically significant associations between the incidence of $\mathrm{T} 1 \mathrm{MI}$ and typical chest pain (odds ratio [OR], 4.40; confidence interval [Cl], 1.46-13.24; $\mathrm{P}=0.008)$, troponin I $(\mathrm{OR}, 1.50 ; \mathrm{Cl}, 1.19-1.90 ; \mathrm{P}<0.001)$, cholesterol levels ( $\mathrm{OR}, 1.01 ; \mathrm{Cl}, 1.00-1.02 ; \mathrm{P}=0.008)$, and $\mathrm{D}$-dimer levels $(\mathrm{OR}, 0.87 ; \mathrm{Cl}, 0.77-0.98 ; \mathrm{P}=0.027)$ (Table 3$)$.

\section{DISCUSSION}

We excluded patients with $\mathrm{T} 3 \mathrm{MI}, \mathrm{T} 4 \mathrm{MI}$, and $\mathrm{T} 5 \mathrm{Ml}$ as we were unable to review the charts of these patients, either because they were dead on arrival to the emergency department or had already undergone an intervention such as $\mathrm{PCl}$ or bypass. T2MI is typified by an imbalance between myocardial oxygen demand and oxygen supply, caused by coronary artery spasm, coronary embolism, anemia, arrhythmias, hypertension, or hypotension. However, many patients with pulmonary disease, anemia, septicemia, renal failure, stroke, tachycardia, or hypotension may have elevated troponin levels. ${ }^{7}$ To the best of our knowledge, this is one of the first studies examining the use of clinical risk factors as a tool for the differential diagnosis of $\mathrm{T} 1 \mathrm{Ml}$ and $\mathrm{T} 2 \mathrm{Ml}$ in patients with high troponin levels.

It has always been challenging for emergency room physicians to differentiate $\mathrm{T} 1 \mathrm{Ml}$ and $\mathrm{T} 2 \mathrm{Ml}$ in patients with elevated troponin
I levels. This study selected factors to determine the best treatment strategy for patients with elevated troponin I levels, and investigated possible correlations among the selected factors. ${ }^{4}$ Most previous studies investigating MI involved patients presenting to the clinic with chest pain. However, this study involved patients with elevated troponin I levels who presented with atypical symptoms or even asymptomatic Ml. Therefore, we believe that this study will be highly useful for managing the patients with $\mathrm{MI}$ in a clinical ED setting.

In this study, there was no significant difference in traditional risk factors such as age, medical history (excluding MI history) and BMI between T1M1 and T2MI patients. $69.2 \%$ of patients with $\mathrm{T} 1 \mathrm{MI}$ were male, and there was a significant difference in the proportion of patients with MI history between the two groups, with 20\% in the T1Ml group and 9.5\% in the T2Ml group. However, results of a multivariate analysis after controlling for confounding variables revealed no significant correlation between sex or $\mathrm{Ml}$ history with either type of $\mathrm{Ml}$, suggesting that it would be difficult to use these factors as tools for differential diagnosis. ${ }^{9}$

In blood test findings, levels of the cardiac biomarkers troponin $I$ and $\mathrm{CK}-\mathrm{MB}$, and the $\mathrm{CK} / \mathrm{CK}-\mathrm{MB}$ ratio were significantly higher in the T1MI group (all $\mathrm{P}<0.001$ ). Previous studies have demonstrated that use of CK as a prognostic marker has a low specificity; the results of this study also revealed no significant difference in $\mathrm{CK}^{4}{ }^{4}$ Our data are consistent with the results of previous studies demonstrating that cardiac biomarkers (especially troponin I) could improve the early diagnosis of MI. ${ }^{10}$ Moreover, this study also demonstrated that the cardiac biomarker test results were highest in the T1Ml group. ${ }^{5}$

The relationship between inflammatory markers and non-ST elevation-acute coronary syndrome has been studied over the past several decades; however, the reasons for the elevated levels of inflammatory markers in non-ST elevation-acute coronary syndrome remain unclear. ${ }^{11}$ Myocardial injury acts as a major inflammatory stimulus and, in turn, acute inflammation can cause myocardial injury, leading to an elevation in CRP levels. ${ }^{4,11}$ However, CRP elevation is a nonspecific phenomenon in the acute phase, and thus, is difficult to use for diagnosis. In our study, CRP levels were found to be elevated in both the T1Ml and T2Ml groups, in agreement with previous studies. ${ }^{12}$ We also looked at the neutrophil counts in each group. Neutrophils are inflammatory mediators, and the inflammatory responses of neutrophils in vulnerable plaques are thought to cause acute coronary syndrome (ACS). Activated leukocytes, including neutrophils, are also found in unstable angina; however, it is not clear whether activated leukocytes are a risk factor for stenosis. ${ }^{11}$ Multivariate analysis correct- 
ed for confounding variables revealed that CRP and neutrophil counts were not significantly associated with either $\mathrm{T} 1 \mathrm{MI}$ or T2Ml, further suggesting that they are not suitable tools for differential diagnosis.

D-dimer is the primary degradation by-product of cross-linked fibrins and is a direct marker of ongoing coagulation coupled with fibrinolysis. ${ }^{13} \mathrm{D}$-dimer levels are elevated in unstable angina and acute MI. ${ }^{14,15}$ However, D-dimer assays typically have low specificity and a wide variation in sensitivity. ${ }^{13}$ The results of this study revealed a valid difference in low D-dimer levels, and we believe that D-dimer levels can be used as a tool to differentiate T1MI from T2MI.

Several studies have investigated the relationship between coronary artery diseases and hypercholesterolemia. Hypercholesterolemia is a well-established causal factor for $\mathrm{Ml}$ and is an atherogenic factor. ${ }^{16}$ In our study, cholesterol levels were found to be significantly higher in the T1Ml group than in the T2Ml group.

The HEART score is used to improve the stratification of the risk of chest pain causes in the emergency department. ${ }^{17}$ However, the HEART score is based on the outcome of major adverse cardiac events, and is helpful in evaluating the risk for ACS and hospitalization in patients with chest pain, but limited in its use as a differential diagnosis tool for determining treatment strategy. When we compared our results with those from the HEART score, we also found that there was no difference in age between $\mathrm{T} 1 \mathrm{Ml}$ and T2Ml patients, although there was a difference in the proportion of each sex between the two groups. In this study, in which patients with ST elevation on ECG were excluded, 65 (28\%) patients were diagnosed with T1MI. Meanwhile, there were no significant differences between T1MI and T2Ml patients with respect to hypertension, diabetes, history of smoking, BMI, and a family history of vascular-related diseases, which are other known risk factors.

Currently, accurate differential diagnosis of $\mathrm{T} 1 \mathrm{Ml}$ and T2MI is important for making decisions regarding treatment methods and discharge. ${ }^{18}$ Previous retrospective studies involving patients with ACS have identified risk factors for ACS, and the findings have been studied for differential diagnosis. Several previous studies over the past few years have attempted to examine various risk factors and combine blood test results so as to differentiate between $\mathrm{T} 1 \mathrm{Ml}$ and $\mathrm{T} 2 \mathrm{Ml}$; however, it has been difficult to definitively determine which factors are useful for making a differential diagnosis. The results of this study revealed significant differences in typical cardiac symptoms, cholesterol levels, and D-dimer levels between $\mathrm{T} 1 \mathrm{Ml}$ and $\mathrm{T} 2 \mathrm{Ml}$ patients. In agreement with other studies, we found that troponin I levels were high in the T1MI group, and there was a significant difference in troponin I levels between $\mathrm{T} 1 \mathrm{Ml}$ and $\mathrm{T} 2 \mathrm{Ml}$ patients, indicating the potential for troponin levels as a tool for differential diagnosis. ${ }^{5}$ We believe that the findings of this study will help to differentiate between T1MI and T2MI in the clinical setting. However, future studies using accumulated data from larger-scale, multicenter trials are required to determine the cut-off values for these diagnostic markers.

This study had several limitations, among which were its single-center design and relatively small sample size; as such, it is difficult to extrapolate our results to the population as a whole. Moreover, this study used retrospective analysis through reviewing patient medical records, and patients and their caregivers were asked to answer questions regarding their medical history, including their history of hyperlipidemia or peripheral arterial disease. Thus, there may have been some omissions from the finished questionnaires. Another limitation was the possibility of selection bias; this study was conducted within the same population at consecutive time intervals. In our analysis of the NT proBNP, several study group patients were not available for initial blood laboratory tests for several reasons. Therefore, the results of this test was not reliable. Finally, in patients with acute coronary syndrome who experience a worsening of the disease, there may be a gradual elevation in cardiac markers and other test findings, whereas in patients with acute coronary syndrome who do not experience any further worsening, there may be a decrease in the levels of cardiac biomarkers. Therefore, there is a possibility of false negative diagnoses depending on when the tests are performed.

In summary, this study analyzed the factors that distinguish T1Ml from T2MI. We found that typical chest pain, high troponin I, high cholesterol and low D-dimer were associated with T1MI rather than T2MI. We believe that these findings will help distinguish between $\mathrm{T} 1 \mathrm{MI}$ and $\mathrm{T} 2 \mathrm{MI}$ and provide appropriate treatment.

\section{CONFLICT OF INTEREST}

No potential conflict of interest relevant to this article was reported.

\section{REFERENCES}

1. Statistics Korea. Cause of death statistics in 2017 [Internet]. Daejeon: Statistics Korea; 2018 [cited 2019 Jul 1]. Available from: http://kostat.go.kr/portal/eng/pressReleases/1/index. board? bmode $=$ readEtaSeq $=371140$.

2. Davies MJ. The pathophysiology of acute coronary syndromes. Heart 2000;83:361-6. 
3. Thygesen $K$, Alpert JS, Jaffe AS, et al. Third universal definition of myocardial infarction. Circulation 2012;126:2020-35.

4. Wright RS, Anderson JL, Adams CD, et al. 2011 ACCF/AHA focused update of the Guidelines for the Management of Patients with Unstable Angina/Non-ST-Elevation Myocardial Infarction (updating the 2007 guideline): a report of the American College of Cardiology Foundation/American Heart Association Task Force on Practice Guidelines developed in collaboration with the American College of Emergency Physicians, Society for Cardiovascular Angiography and Interventions, and Society of Thoracic Surgeons. J Am Coll Cardiol 2011;57:192059.

5. Reichlin T, Hochholzer W, Bassetti S, et al. Early diagnosis of myocardial infarction with sensitive cardiac troponin assays. N Engl J Med 2009;361:858-67.

6. Meigher S, Thode HC, Peacock WF, Bock JL, Gruberg L, Singer AJ. Causes of elevated cardiac troponins in the emergency department and their associated mortality. Acad Emerg Med 2016;23:1267-73.

7. Six $A J$, Cullen $L$, Backus BE, et al. The HEART score for the assessment of patients with chest pain in the emergency department: a multinational validation study. Crit Pathw Cardiol 2013;12:121-6.

8. Task Force for Diagnosis and Treatment of Non-ST-Segment Elevation Acute Coronary Syndromes of European Society of Cardiology, Bassand JP, Hamm CW, et al. Guidelines for the diagnosis and treatment of non-ST-segment elevation acute coronary syndromes. Eur Heart J 2007;28:1598-660.

9. Fox WR, Diercks DB. Troponin assay use in the emergency department for management of patients with potential acute coronary syndrome: current use and future directions. Clin Exp Emerg Med 2016;3:1-8.
10. Buffon A, Biasucci LM, Liuzzo G, D'Onofrio G, Crea F, Maseri A. Widespread coronary inflammation in unstable angina. N Engl J Med 2002;347:5-12.

11. Oh J, Kim SH, Park KN, et al. High-sensitivity C-reactive protein/albumin ratio as a predictor of in-hospital mortality in older adults admitted to the emergency department. Clin Exp Emerg Med 2017;4:19-24.

12. Shitrit D, Bar-Gil Shitrit A, Rudensky B, Sulkes J, Gutterer N, Zviony D. Role of ELISA D-dimer test in patients with unstable angina pectoris presenting at the emergency department with a normal electrocardiogram. Am J Hematol 2004;77:147-50.

13. Moss AJ, Goldstein RE, Marder VJ, et al. Thrombogenic factors and recurrent coronary events. Circulation 1999;99:2517-22.

14. Corrigan D, Prucnal C, Kabrhel C. Pulmonary embolism: the diagnosis, risk-stratification, treatment and disposition of emergency department patients. Clin Exp Emerg Med 2016;3:11725.

15. Sakata K, Miho N, Shirotani M, Yoshida H, Takada Y, Takada A. Remnant-like particle cholesterol is a major risk factor for myocardial infarction in vasospastic angina with nearly normal coronary artery. Atherosclerosis 1998;136:225-31.

16. Martin SS, Faridi KF, Joshi PH, et al. Remnant lipoprotein cholesterol and mortality after acute myocardial infarction: further evidence for a hypercholesterolemia paradox from the TRIUMPH registry. Clin Cardiol 2015;38:660-7.

17. Nowak RM, Jacobsen G, Christenson RH, Moyer M, Hudson M, McCord J. Differentiating type 1 and 2 acute myocardial infarctions using the $\mathrm{N}$-terminal pro B-type natriuretic peptide/ cardiac troponin T ratio. Am J Emerg Med 2018;36:1849-54.

18. Kuo DC, Peacock WF. Diagnosing and managing acute heart failure in the emergency department. Clin Exp Emerg Med 2015;2:141-9. 